\title{
NEIGHBORHOODS OF POINTS IN CODIMENSION-ONE SUBMANIFOLDS LIE IN CODIMENSION-ONE SPHERES
}

\author{
FREDRIC D. ANCEL
}

(Communicated by Doug W. Curtis)

\begin{abstract}
For $n \geq 4$, let $M$ be an $(n-1)$-manifold embedded in an $n$ manifold $N$. For each point $p$ of $M$, there is an $(n-1)$-sphere $\Sigma$ in $N$ such that $\Sigma \cap M$ is a neighborhood of $p$ in $M$.
\end{abstract}

We work in the category of topological manifolds without boundary and topological embeddings.

The $n=3$ case of this result is established by Theorem 5 of [2]. A weak version of this result for $n \geq 5$ is found in Theorem 5 B.10 of [3]. This particular proof arose in response to a private query from D. L. Loveland of Utah State University.

This type of result is used to generalize theorems concerning local properties of wild codimension-one spheres into theorems about arbitrary wild codimension-one submanifolds. One application is found in Theorem 6 of [2]. Another application is discussed on pages 38 and 39 of [1].

PROOF. Without loss of generality, we can cut $M$ and $N$ down to assure that both are orientable. This makes any embedding of $M$ in $N 2$-sided. Now, for an open subset $V$ of $M$, an embedding $e: V \rightarrow N$ is tame if there is an embedding $E: V \times \mathbf{R} \rightarrow N$ such that $E(x, 0)=e(x)$ for each $x \in V$; the embedding $E$ is called a collar of $e$.

Let $\left\{U_{i}: i \geq 0\right\}$ be a decreasing sequence of open neighborhoods of $p$ in $N$ with diameters converging to zero. Let $\left\{D_{i}: i \geq 0\right\}$ be a sequence of $(n-1)$-balls in $M$ such that for each $i \geq 0,\{p\} \cup D_{i+1} \subset \operatorname{int}\left(D_{i}\right)$ and $D_{i} \subset U_{i}$. For $0 \leq i<j<\infty$, let $A(i, j)=\left(\operatorname{int}\left(D_{i}\right)\right)-D_{j}$ and let $A(i, \infty)=\left(\operatorname{int}\left(D_{i}\right)\right)-\{p\}$.

Let $e_{0}: M \rightarrow N$ denote the given inclusion. Repeated applications of Theorem 2.2 of [1] yields a sequence of embeddings $e_{i}: M \rightarrow N$ which, for each $i \geq 1$, satisfy the following three conditions.

(1) $e_{i}=e_{i-1}$ on $M-A(i-1, i+1)$.

(2) $e_{i} \mid A(0, i+1)$ is tame.

(3) $e_{i}\left(D_{j}\right) \subset U_{j}$ for each $j \geq 0$.

It follows that the sequence $\left\{e_{i}\right\}$ converges to an embedding $f: M \rightarrow N$ such that for each $i \geq 0, f=e_{i}$ on $M-A(i, \infty)$. Consequently, $f \mid A(0, \infty)$ is tame.

According to [4], $f$ cannot have isolated wild points. Hence, $f \mid \operatorname{int}\left(D_{0}\right)$ is, in fact, tame. Thus, $f \mid \operatorname{int}\left(D_{0}\right)$ has a collar, which we use to slide $f$ to an embedding $g: M \rightarrow N$ such that $g\left(\operatorname{int}\left(D_{0}\right)\right) \cap f\left(\operatorname{int}\left(D_{0}\right)\right)=\varnothing$ and $g=f$ on $M-\operatorname{int}\left(D_{0}\right)$.

As $p=f(p) \in f\left(\operatorname{int}\left(D_{0}\right)\right)$, there is an $i \geq 0$ such that $g(M) \cap U_{i}=\varnothing$. Since $e_{i}\left(D_{i}\right) \subset U_{i}$ and $e_{i}=f$ on $M-\operatorname{int}\left(D_{i}\right)$, then $e_{i}\left(\operatorname{int}\left(D_{0}\right)\right) \cap g\left(\operatorname{int}\left(D_{0}\right)\right)=\varnothing$ and

Received by the editors July 27, 1987.

1980 Mathematics Subject Classification (1985 Revision). Primary 57N35, 57N45. 
$e_{i}=g$ on $\partial D_{0}$. So $\Sigma=e_{i}\left(D_{0}\right) \cup g\left(D_{0}\right)$ is an $(n-1)$-sphere. Since $e_{i}=e_{0}$ on $D_{i+1}$, then $D_{i+1} \subset \Sigma \cap M$.

\section{REFERENCES}

1. F. D. Ancel, Resolving wild embeddings of codimension-one manifolds in manifolds of dimensions greater than 3, Topology Appl. 24 (1986), 13-40.

2. R. H. Bing, A surface is tame if its complement is 1-ULC, Trans. Amer. Math. Soc. 101 (1961), 294-305.

3. R. J. Daverman, Embeddings of $(n-1)$-spheres in Euclidean $n$-space, Bull. Amer. Math. Soc. 84 (1978), 377-405.

4. R. C. Kirby, On the set of non-locally flat points of a submanifold of codimension one, Ann. of Math. (2) 88 (1968), 281-290.

Department of Mathematical Sciences, UNiversity of Wisconsin-MilWaUKEE, WISCONSIN 53201 